Ebisu Ebisu

Études japonaises Études japonaises

53 | 2016

1914-1918, une guerre mondiale ? La perspective japonaise

\title{
Les émeutes du riz de 1918 : le grand tournant
}

大転機となった1918年の米騷動

The Rice Riots of 1918: A Historic Watershed

\section{Pierre-François Souyri}

URL : https://journals.openedition.org/ebisu/1868

DOI : 10.4000/ebisu. 1868

ISSN : 2189-1893

Éditeur

Institut français de recherche sur le Japon à la Maison franco-japonaise (UMIFRE 19 MEAE-CNRS)

Édition imprimée

Date de publication : 10 décembre 2016

Pagination : 101-128

ISSN : 1340-3656

Référence électronique

Pierre-François Souyri, «Les émeutes du riz de 1918 : le grand tournant », Ebisu [En ligne], 53 | 2016, mis en ligne le 10 décembre 2016, consulté le 08 novembre 2021. URL : http://

journals.openedition.org/ebisu/1868 ; DOI : https://doi.org/10.4000/ebisu.1868 


\section{Les émeutes du riz de 1918}

Le grand tournant

\section{Pierre-François SouYrI}

ピエール＝フランソワ・スイリ

\ots-clés : Émeutes, inflation, guerre, démocratie, misère, histoire sociale.

L'auteur : Pierre-François Souyri est professeur d'histoire du Japon à l'université de Genève. Il s'est notamment intéressé à l'histoire des mouvements populaires et est l'auteur de nombreux articles et ouvrages, dont récemment : Moderne sans être occidental. Aux origines du Japon d'aujourd'hui (Gallimard, 2016).

Résumé : Au cours de l'été 1918, le Japon est confronté à une flambée de violences, les émeutes du riz. Après avoir retracé le contexte politique, économique et social de l'époque à l'issue de la guerre, alors que le pays s'apprête à intervenir en Sibérie, l'auteur s'attache à décrire les manifestations concrètes de ces émeutes: l'inflation déclenche la colère des populations, les femmes des ports de la mer du Japon d'abord, avant de toucher presque l'ensemble du pays. Quelles forces sociales entrèrent-elles en action? Pourquoi? Comment ces émeutes furent-elles analysées par les commentateurs de l'époque? Quel est leur sens profond? Dernières émeutes d'Ancien Régime ou annonce d'une conflictualité sociale nouvelle? 
マキーワード

暴動、インフレ、戦争、民主主義、貧困、 社会の歴史

\section{著者}

ピエール＝フランソワ・スイリはジュネー ヴ大学で日本史の教鞭をとる。とりわけ日 本における市民運動の歴史を研究対象とす る。多数の著書があり、近著には『Moderne sans être occidental, aux origines du Japon d'aujourd'hui』(Gallimard, 2016) がある。

\section{要旨}

1918 年夏の間、暴力の激化した米騒動が起 こった。筆者は、シベリア出兵をひかえた日 本の第一次世界大戦後の政治的、経済的、社 会的背景をなぞり、これらの暴動におけるデ モが具体的にどのような形を取ってきたかを 明らかにした。日本海の港の女沖仕をはじめ とする市民の怒りが膨らみ、後にほぼ日本全 土で米騒動が起こった。どのような社会的な 力が市民を突き動かしたのか？それはなぜ か? 当時の知識人たちの反応は? 今日どのよ うに解釈できるか? 旧体制最後の暴動か、そ れとも、新しい社会の紛争状態の兆しか?
\ Keywords: Riots, Inflation, War, Democracy, Poverty, Social History.

The Author: Pierre-François Souyri is a professor in Japanese history at the University of Geneva. Previous research has focused on the history of popular movements in Japan. He has published numerous articles and books, including Moderne sans être occidental, aux origines du Japon d'aujourd'hui (Gallimard, 2016).

Abstract: In the summer of 1918 Japan was hit by a wave of violent protests: the rice riots. After re-examining the social, political and economic context of the time, with WWI over and Japan poised to take part in the Siberian Intervention, the author seeks to describe the concrete form these riots took: the inflation that enraged the Japanese people, starting with women in coastal towns along the Sea of Japan, before spreading across the country. What social forces were at work and why? How were the riots analysed by commentators of the day? What was their deeper meaning? Were they the last riots of the Old Regime or the sign of a new form of social conflict? 


\section{Les émeutes du riz de 1918 Le grand tournant}

Pierre-François SOUYRI*

Les " émeutes du riz » qui touchèrent le pays durant l'été 1918 sont sans doute le plus grand soulèvement populaire qu'ait connu le Japon au cours de son histoire. Le mouvement concerna 38 villes, 153 bourgs et 77 villages, selon des rapports sans doute sous évalués, entraînant environ 700000 personnes, peut-être plus encore, dans des manifestations et des cortèges de rue dont certains tournèrent à l'affrontement avec la police ou l'armée ${ }^{1}$. Ces émeutes sonnèrent comme un coup de tonnerre dans un pays où l'agitation politique et sociale était non pas inexistante mais somme toute mesurée. Elles furent très vite comprises comme un mouvement de nature ambiguë, à la fois une révolte du type de celles qui avaient éclaté sous l'ancien régime Tokugawa et dans les premières années $\mathrm{Meiji}^{2}$, un $i k k i$ 一揆, et dans ce cas, le plus important mais aussi - on le sait aujourd'hui - le dernier d'entre eux. On y vit aussi un mouvement de colère populaire, alliant aux anciens modes de protestation des revendications touchant aux conditions d'existence, de travail et aux salaires. Quoi qu'il en soit, les contemporains constatèrent que les émeutes du riz apparaissaient comme un tournant dans l'histoire politique et sociale du Japon au début du $\mathrm{Xx}^{\mathrm{e}}$ siècle, déclenchant

1. Inoue \& Watanabe (1962: 496).

2. Sur les $i k k i$, voir Katsumata (2011 [1982]).

* Université de Genève. 
dans leur foulée une vague de protestations, notamment dans la classe ouvrière, le tout dans un contexte international de montée de l'agitation sociale au lendemain de la révolution russe.

La répression qui frappa au Japon le mouvement socialiste et plus généralement le mouvement ouvrier à partir de $1907^{3}$, puis la vague d'arrestations de 1910 suivie d'exécutions en janvier 1911 dans le cadre de l'affaire du complot de lèse-majesté (taigyaku jiken 大逆事件) ${ }^{4}$, désorganisèrent, voire décapitèrent, un mouvement social naissant. Le jeune poète Ishikawa Takuboku 石川啄木 (1886-1912) évoqua alors en une formule qui devint célèbre une "période de repli ", " une période bloquée " ${ }^{5}$. Outre la répression proprement dite et la surveillance policière incessante, la crainte d'être mêlé de près ou de loin à un complot contre l'empereur éloignait les sympathisants de la cause socialiste, isolant encore un peu plus les militants. Cet isolement fut d'ailleurs aggravé par la scission des partis socialistes européens qui, presque tous, rallièrent la cause nationale pendant la Première Guerre mondiale. Entre 1910-1911 et 1917-1918, le mouvement socialiste d'un côté, et le mouvement ouvrier syndical de l'autre, connurent ce qui au Japon est qualifié d' "ère d'hiver" ou de " période d'hibernation " (fuyu no jidai 冬の時代). La tendance générale était au repli. Les rares militants étaient arrêtés pour simple distribution de tracts et la démoralisation les gagnait ${ }^{6}$.

Pourtant, l'agitation continua dans les années 1913-1918. Mais il s'agissait d'une agitation politique qui touchait plutôt les nouvelles couches moyennes urbaines, avec le développement d'un mouvement en faveur du

3. À la suite des mouvements de grève qui atteignirent un nombre record en 1907, la police procéda à des arrestations dans les milieux ouvriers et syndicalistes.

4. À partir du printemps 1910, des rafles policières aboutirent à plus d'une centaine d'arrestations parmi les militants socialistes, suite à l'accusation d'un complot visant l'empereur dont les preuves ne seront jamais produites. 26 militants furent jugés, 24 condamnés à mort et 12 exécutés, dont Kōtoku Shūsui 幸徳秋水 (1871-1911) l’un des principaux leaders du mouvement socialiste radical. Sur cette affaire, en français, voir notamment Lévy (2002, 2010), ainsi que Souyri (2016).

5. En août 1910, Ishikawa Takuboku, proche des milieux socialistes, proposa au journal Tōkyō Asahi shinbun 東京朝日新聞 (qui le refusa) un essai intitulé "La période actuelle est bloquée " (Jidai heisoku no genjō 時代閉塞の現状). Le texte fut finalement publié en 1913. Réédité dans Ishikawa (1969 : 467-480).

6. Arahata (2016 [1975] : 383). 
suffrage universel (masculin) en remplacement du suffrage censitaire élitiste, et cherchant à promouvoir un gouvernement représentant les forces parlementaires et non les anciennes factions féodales. Certains libéraux voyaient dans le changement de règne impérial en 1912 l'avènement sinon d'une nouvelle politique, du moins d'une nouvelle génération.

\section{Le contexte politique : de la nature de la démocratie}

En 1912, le cabinet Saionji, sous l'influence du parti Seiyūkai 政友会, refusa, pour des raisons budgétaires, la création des deux nouvelles divisions réclamées par l'armée de terre pour le maintien de l'ordre en Corée. Le ministre de la Guerre démissionna mais l'armée de terre refusa d'en désigner un nouveau ${ }^{7}$ : ce fut la première épreuve de force entre un cabinet civil et l'étatmajor. Saionji Kinmochi 西園寺公望 (1849-1940) fut alors contraint de démissionner. Katsura Tarō 桂太郎 (1848-1913) forma un cabinet conservateur en s'appuyant sur les forces traditionnelles, les anciennes cliques féodales (hanbatsu 藩閥) des fiefs du Sud-Ouest, la bureaucratie et l'état-major des deux armes. Mais une partie de la presse se déchaîna contre ce retour en force des milieux les plus traditionnels. L'opposition se mobilisa avec des mots d'ordre du type "Protégeons le régime constitutionnel ! ", " À bas les cliques féodales!». Certains milieux financiers prêtèrent de l'argent aux députés et aux journalistes d'opposition pour déclencher une dynamique susceptible de faire tomber le gouvernement Katsura, fraîchement mis en place. Ce dernier menaça d'user de la censure et de la répression mais, début 1913, des manifestations populaires contre le gouvernement empêchèrent la Diète de siéger, tandis que des locaux de police et des sièges de journaux étaient incendiés. Après 53 jours de gouvernement, Katsura démissionna devant le risque de débordement général. Le coup de force de l'armée avait échoué.

La démission de Katsura sonna le coup d'envoi de la vague démocratique dite de Taishō. À titre d'exemple, à Namerikawa, sur la côte de la mer du

7. Les ministres de la guerre et de la marine ne pouvaient être issus que des cadres de l'armée de terre et de la marine. Sans accord de l'état-major, il ne pouvait donc y avoir de ministre. 
Japon, l'un des bourgs où débuteront les émeutes de 1918, 800 personnes vinrent écouter le 21 juin 1913 les orateurs du mouvement qui dénonçaient les cliques féodales monopolisant le pouvoir et parlaient en faveur du suffrage universel ${ }^{8}$. Ce mouvement pour le suffrage universel (masculin), fusen undō 普選運動, gagna sans cesse en importance après 1913 et pourtant le gouvernement ne céda qu'en $1925^{\circ}$. Dès 1914, par un curieux effet de retournement, la propagande en faveur de la guerre contre l'Allemagne reprit le thème de la lutte contre le militarisme allemand au nom des idéaux démocratiques proclamés par les alliés. La propagande belliciste offrit ainsi au mouvement démocratique une nouvelle raison d'espérer...

Parmi les porte-paroles de ce mouvement, un professeur de l'université impériale de Tokyo, Yoshino Sakuzō 吉野作造 (1878-1933), inventa un discours particulier sur la démocratie, la démocratie de type minpon shugi 民本主義, démocratie au sein du régime impérial. Pour traduire le mot de démocratie, on parlait au Japon à la fin du XIX siècle de minshu shugi 民主主義 (doctrine où le peuple est son propre maître, doctrine socialisante et dangereuse, nous dit Yoshino ${ }^{10}$ ) ou de shumin shugi 主民主義 (doctrine où le maître est le peuple). On a également pu évoquer minshū shugi 民衆 主義 (doctrine des masses populaires) et même heimin shugi 平民主義 (doctrine du peuple). C'est finalement le premier terme qui va l'emporter, et passer aussi en chinois (mínzhŭ zhŭ yì), en coréen (minju juŭi) et même en vietnamien (dânchủ chủ nghĩa). Yoshino avance de son côté l'expression de minpon shugi (doctrine où le peuple est au fondement des choses), dont il nous explique qu'elle est la traduction en japonais du mot occidental "démocratie ${ }^{11}$. Tous ces termes constituèrent les mots-clés des discours politiques libéraux au cours de l'ère Taishō (1912-1926). Minpon shugi, même s'il se retrouve dans de rares occurrences chez Mencius pour désigner la politique, est quasi inexistant en chinois et très peu usité en coréen.

8. Kamiya (1986:1).

9. Des féministes s'engagèrent de leur côté, non sans résultats, pour le suffrage universel réel et lancèrent un mouvement qui, ironiquement, s'intitulait lui aussi fusen undō 婦選運動 (mouvement pour le droit de vote des femmes, le premier caractère ayant été changé). Celui-ci faillit obtenir satisfaction au début des années 1920, mais le Parlement rejeta finalement la proposition, à quelques voix près.

10. Yoshino (1984 [1916] : 113).

11. Yoshino (1984 [1916] : 110). 
Yoshino se fit vraiment remarquer quand, déjà professeur à l'université, il publia un long article dans la revue Chūō kōron 中央公論, en janvier 1916, intitulé " Les principes du gouvernement constitutionnel et les moyens de les réaliser pleinement ${ }^{12} »$. Dans cet article, il évoquait pour la première fois son idée de minpon shugi, de "démocratie à la japonaise $»^{13}$. Le concept de minpon shugi - mot aujourd'hui quelque peu démodé - résonne fortement car il associe empereur, nation et démocratie, c'est-à-dire qu'il opère une forme de synthèse entre les tenants de l'autorité impériale, les thuriféraires de la nation et les classes moyennes qui revendiquent une participation plus active aux décisions politiques. Pour Yoshino, ce concept relève de la science politique moderne. Il pose la question : comment faire progresser les idées et les pratiques démocratiques dans le cadre de la constitution impériale ? Pour lui, minpon shugi est non seulement la traduction du concept occidental de démocratie mais a aussi pour signification le fait que « les objectifs fondamentaux de l'activité souveraine de l'État doivent résider dans le peuple ${ }^{14} »$. Selon Yoshino, un régime constitutionnel ne peut se limiter à l'application stricte de la Constitution, mais doit se consacrer à la mise en pratique de l'esprit qui a présidé à la mise en place de ladite Constitution. Et quel est donc cet esprit ? Yoshino répond : ce sont les trois principes fondamentaux de la protection des droits du peuple, de la séparation des trois pouvoirs (exécutif, législatif et judiciaire), du système d'élection des représentants du peuple. L'élargissement réel des droits du peuple est possible, selon lui, dans le cadre de la Constitution.

Pour Yoshino, le fondement spirituel de la Constitution, c'est la démocratie en tant que telle. La politique n'a pour objet que le bonheur de la population ordinaire et toute décision politique doit se situer dans ce cadre $^{15}$. Assurer la protection du peuple, respecter la séparation des pouvoirs exécutif, législatif et judiciaire, élire des assemblées au suffrage universel, tels doivent être les principes ultimes d'un gouvernement constitutionnel. Pour cela, il est nécessaire d'assurer le respect de la liberté d'expression et

12. Yoshino (1984 [1916]).

13. Le mot circulait déjà et avait été utilisé par plusieurs journalistes avant que Yoshino n'en donne une définition plus structurée.

14. Yoshino (1984 [1916] : 93).

15. Yoshino (1984 [1916] : 121). 
d'élargir le suffrage censitaire en vigueur. Yoshino oppose « la population ordinaire " aux « classes privilégiées », c'est-à-dire l'aristocratie qui bénéficie de sa position historique et les capitalistes qui ont profité de la conjoncture récente. Contre «les partisans de l'invasion, le militarisme, le capitalisme, les tenants des privilèges de classe », il préconise «la domination de la sagesse et de la morale du peuple " (kokumin chitoku 国民知徳), “ un principe immuable qui devrait dominer le monde ». Une démocratie réelle au Japon permettrait, à son avis, "d'avancer avec le reste du monde ", mais cette avancée reste liée au niveau général d'éducation du peuple. Il termine en proclamant haut et fort la nécessité d'un système fondé sur le gouvernement des partis politiques élus au Parlement au suffrage universel, la suprématie de la Chambre basse sur la Chambre des pairs, la responsabilité du cabinet devant le Parlement ${ }^{16}$, et se prononce en faveur de réformes démocratiques dans le cadre de la Constitution impériale.

Il n'est pas inintéressant à ce stade de remarquer qu'à côté d'un vocabulaire très influencé par la philosophie politique occidentale, Yoshino, et d'autres démocrates dans son sillage, utilisent dans leurs travaux une rhétorique largement tirée des classiques chinois. À partir d'expressions forgées autour de quatre idéogrammes comme kanmin dōkyō 官民同共 (coopération du peuple et des fonctionnaires), kunmin dōchi 君民同治 ou kunmin dosei 君民同政 (gouvernement conjoint du souverain et du peuple), banmin dōchi 万民同治 (gouvernement conjoint de tout le peuple), expressions issues du répertoire chinois, ils tentent de faire passer l'idée d'un processus engendré par des notions traditionnelles, comme si ces tournures avaient valeur intégrative en recréant du connu et du commun ${ }^{17}$. Ces conceptions reposent finalement sur un mélange de foi dans le progrès mais aussi de volonté très influencée par la pensée classique de faire triompher la morale et l'harmonie sociale.

Dès leur parution, les thèses de Yoshino furent critiquées par des socialistes qui lui reprochèrent d'abandonner certains principes essentiels, de couper l'idée de démocratie en tranches, d'en réduire le sens. Ils l'accusèrent

16. Yoshino (1984 [1916] : 179-180). À propos du droit de vote pour les femmes, Yoshino reste assez ambigu même s'il encourage l'existence de mouvements féministes (Yoshino 1995 [1915]).

17. Duus et Scheiner (1998: 174). 
de ne vouloir que le développement de pratiques démocratiques dans le cadre d'un État dominé par l'empereur: le minpon shugi serait ainsi une sorte de "démocratie impériale ", une démocratie à l'intérieur d'un système qui, par essence, est non démocratique. Yoshino aurait renoncé en fait à l'idée démocratique d'" un gouvernement du peuple pour le peuple par le peuple ", selon la fameuse expression de Lincoln. Plus grave, il aurait abandonné au profit de l'institution impériale l'idée d'une souveraineté populaire $^{18}$.

L'historien Narita Ryūichi nuance cependant ces critiques : Yoshino était un pragmatique qui savait la répression toujours possible. Il aurait en fait tenté un compromis pour vider de leur substance les tendances autoritaires manifestées dans le texte constitutionnel. En clair, il n'était pas en désaccord avec les tenants d'une conception plus radicale de la démocratie, mais était plus attaché aux réformes à venir de la Constitution qu’à sa critique théorique $^{19}$. Yoshino voulait faire savoir aux vieux conservateurs que la "démocratie" (minpon shugi) ne menaçait pas le corps national, le kokutai 国体. Le drame de Yoshino néanmoins, c'est qu'il fut en avance sur son temps dans les années 1914-18 et critiqué dès les années 1919-1920 par une nouvelle génération pour sa relative modération ${ }^{20}$. Pourtant sa pensée influença considérablement ses contemporains jusque dans des milieux $a$ priori peu versés dans les débats théoriques de science politique.

\section{Le boom économique de la guerre : profiteurs et misérables}

Au cours des années de guerre, le capitalisme japonais, qui avait achevé sa révolution industrielle dans les premières années du $\mathrm{xx}^{\mathrm{e}}$ siècle, accomplit un véritable bond en avant ${ }^{21}$. Profitant des difficultés des Alliés en guerre, le Japon imposa ses produits sur les principaux marchés d'Asie, notamment la Chine et, pour la première fois, exporta à destination de l'Europe certains

18. Yamakawa (1976 [1918] : 15-35).

19. Narita $(2007: 31)$.

20. Stegewerns (2003: 127).

21. Pour un indice de la production industrielle (indice Nagoya koshō) on passe de 100 en 1914 à 487 en 1919. 
produits métallurgiques et miniers ainsi que des fournitures militaires. Le poids de l'industrie lourde s'accrut tandis que naissait une puissante industrie chimique. C'est le moment où apparurent des conglomérats, les zaibatsu 財閥, comparables par leur taille aux grandes entreprises américaines, allemandes ou britanniques. Entre 1914 et 1918, on estime que le PNB s'accrut de $40 \%$ et que la croissance atteignit $9 \%$ par $a^{22}$. Pour la première fois, le poids des activités industrielles dépassa celui des activités agricoles. Cette activité industrielle donna un coup de fouet aux exportations et au commerce maritime en particulier. Entre 1914 et 1918, les prix doublèrent en moyenne alors que les salaires connurent un décrochage notable, la hausse nominale n'étant que de $50 \%$ environ ${ }^{23}$. Dans l'industrie, les profits s'accumulaient tandis que surgissaient de "nouveaux riches", les narikin 成金, ceux qui avaient su profiter de la croissance rapide pour faire fortune en peu de temps. Ces nouveaux magnats s'enrichirent dans les secteurs industriels les plus divers (pharmacie, teinturerie, métallurgie, papeterie, textile...), mais les fortunes les plus impressionnantes tout autant que soudaines apparurent surtout dans l'exploitation des mines, puis dans le secteur de l'appareillage électrique (Hitachi par exemple) et du transport maritime. Le nombre des ouvriers travaillant dans le secteur moderne de l'industrie doubla entre 1914 et 1919, notamment dans l'industrie lourde et les transports. La nouvelle classe ouvrière était composée désormais de plus en plus de travailleurs masculins adultes et qualifiés, alors que la première industrialisation fondée sur l'industrie légère (textile surtout) avait été le fait d'une main-d'œuvre féminine très jeune et peu qualifiée. On eut donc au Japon, toutes proportions gardées, un phénomène à l'inverse de ce qui se produisit dans les pays d'Europe où la Première Guerre mondiale favorisa plutôt l'essor du travail industriel féminin. Dans le cas japonais, la féminisation du salariat à cette époque toucha plutôt le secteur des services.

Ce développement rapide de l'industrie japonaise dans les années 19141918 fut à l'origine d'un retournement de la conjoncture politique, fruit d'une crise dans les relations du travail : la rareté de la main-d'œuvre qualifiée créa un rapport de force nouveau et relativement favorable aux

22. Crawcour (1997: 101).

23. Imai (1998 [1974] : 105). Sur une base 100 en 1914, les salaires étaient à 115 environ quand les prix des biens de consommation atteignaient 173 (Imoto et al. 2004 : 74). 
travailleurs, qui cherchèrent dès lors à obtenir de meilleurs salaires ${ }^{24}$. Dans un contexte international nouveau - lié principalement à la révolution russe - où les mouvements sociaux touchèrent de nombreux pays d'Europe, le nombre de travailleurs japonais en grève s'accrut en 1917 avant de connaître un premier pic en 1918.

Le nombre des amicales et mutuelles ouvrières, qui jouaient dans les faits le rôle de syndicats non officiels, était en constante augmentation à la fin des années 1910. Suzuki Bunji 鈴木文治 (1885-1946), diplômé de l'université de Tokyo converti au protestantisme et préoccupé de questions sociales, fonda en 1912 la Yūaikai 友愛会 (L’Amicale), conçue comme une société ouvrière de secours mutuel. Cette association, qui ne comptait qu'une quinzaine de membres à ses débuts, mettait l'accent sur le développement harmonieux des relations entre patrons et ouvriers. Suzuki entretenait d'excellentes relations avec Samuel Gompers (1850-1924), le charismatique leader de la Fédération américaine du travail (AFL), qu'il avait rencontré lors d'un séjour aux États-Unis, et il avait dans l'idée de fonder un syndicat ouvrier de type américain, qui chercherait à obtenir des réformes substantielles en négociant des compromis avec le patronat. La Yūaikai reçut le patronage de Shibusawa Eiichi 渋沢栄一 (1840-1931), l'un des capitaines de l'industrie et de la finance les plus en vue de son temps ${ }^{25}$. En 1915, elle comptait plus de 6000 membres, 22000 au printemps $1918^{26}$.

À partir du milieu des années 1910, la question sociale fut en effet l'objet d'une littérature dans les revues et la presse ${ }^{27}$. Dans cette veine, il faut signaler notamment Binbō monogatari 貧乏物語 (Roman de la misère) ${ }^{28}$, composé en livre en 1917, à la suite d'une série d'articles publiés l'année précédente dans l'Ósaka Asahi shinbun 大阪朝日新聞. L'auteur, un économiste qui venait d'être nommé professeur de l'université impériale de Kyoto, Kawakami Hajime 河上肇 (1879-1946), rédigea cet essai à son retour d'un séjour en Europe (1913-1915).

24. Le nombre de mouvements sociaux portant sur l'amélioration des salaires passe de 25 en 1914, à 304 en 1917, et 340 en 1918 (Imoto et al. 2004 : 76). Les salaires réels vont en moyenne doubler entre 1918 et 1922 (ibid.).

25. Hamon (2007 : 234 sqq.).

26. Eguchi (1989: 74-75).

27. Garon (1987: 10-38).

28. Il s'agit en fait, malgré ce titre, d'un essai économique sur la misère. 
Dans l'introduction de son ouvrage, Kawakami écrit : «L'homme ne vit pas seulement de pain, mais il ne vit pas non plus sans pain : c'est en gardant pareille idée en tête que l'auteur de ces lignes a rédigé cet ouvrage ${ }^{29}$ ». Kawakami montre que dans les pays d'Europe les plus développés la pauvreté touche une majorité de la population et qu'au Japon, pays qui a su profiter de la conjoncture née de la Première Guerre mondiale pour développer une économie capitaliste solide, la guerre donne naissance à un groupe social de "nouveaux riches ». Mais cette prospérité a ses ombres, car elle a aussi favorisé l'émergence d'une misère sociale, aggravée par la hausse des prix. Kawakami part d'une constatation terrible : alors que l'économie ne cesse de se développer, le nombre de pauvres, au lieu de reculer, ne fait que s'accroître. Il analyse le phénomène d'un point de vue humaniste, avec le souci de faire prendre conscience au lecteur des lacunes et des insuffisances criantes des systèmes de prévoyance sociale et de solidarité. Kawakami écrit là, sans le vouloir, une sorte de bestseller (120000 exemplaires vendus dès la première édition), un peu à l'image de celui rédigé par Yokoyama Gennosuke 横山源之助 (1871-1915) presque vingt ans plus tôt ${ }^{30}$. "Il est étonnant de voir pareille misère dans des pays civilisés comme les nôtres " écrit Kawakami dans l'introduction de son essai, avec presque de la naïveté $^{31}$. Plus loin, constatant que tous les pays occidentaux sont confrontés à cette même question et qu'en Angleterre, "l'un des pays les plus riches du monde, les pauvres forment encore $30 \%$ de la population ${ }^{32}$ ", il conclut : " la misère est la grande maladie du $\mathrm{xx}^{\mathrm{e}}$ siècle ». Pour guérir cette maladie, il ne voit qu'une seule solution, l'implication active de l'État et « la réforme de l'opinion ${ }^{33}$ ».

Kawakami Hajime s'interroge sur l'absurdité d'un système qu'il juge incapable de se réformer et se rapproche des thèses défendues par les socialistes. Le problème réside, selon lui, dans la demande qui est insuffisante et ne pousse pas le système à produire autant qu'il faut, d'autant que le peu de richesse produite est mal réparti ou redistribué. L'État doit intervenir

29. Kawakami (2008 [1917] : 4).

30. Yokoyama (1985 [1899]). Voir, à ce sujet, Souyri (2016 : 363-368).

31. Kawakami (2008 [1917] : 13).

32. Kawakami (2008 [1917] : 16).

33. Kawakami (2008 [1917] : 163). 
par des lois somptuaires pour obliger les riches à ne pas surconsommer ${ }^{34}$. Kawakami reste dans une logique " asiatique ", c'est-à-dire dans l'idée très confucéenne que l'harmonie sociale est le produit d'une forme de vertu, et que le chaos est le fruit de l'excès. Égoïsme et individualisme conduisent, sous prétexte de liberté, à créer de la pauvreté. C'est donc au nom d'autres principes, plus moraux, que l'ordre peut être rétabli. En fin de compte, ce n'est pas l'économie elle-même mais la vertu, mise en œuvre par l'État, qui peut combattre les désordres de l'économie. Et Kawakami de se montrer finalement partisan d'un "étatisme économique " fondé sur des principes moraux. Il faut bien reconnaître que, jusqu'alors, les économistes ne traitaient souvent que de l'enrichissement ou de l'épargne. Kawakami fut sans doute l'un des premiers universitaires japonais à se pencher sur le phénomène de la pauvreté $e^{35}$. On mesure dans son discours - comme dans celui de Yoshino, nous l'avons vu - la prégnance des références classiques confucéennes à côté de réflexions inspirées par la lecture d'Adam Smith ou de Karl Marx.

La première loi sociale votée en 1911 entra en vigueur en 1916, mais la réputation du travail en usine, assimilé au bagne, restait terrible. Les principales dispositions de la nouvelle loi prévoyaient l'interdiction du travail en usine aux enfants de moins de 12 ans et l'interdiction du travail de nuit (entre $22 \mathrm{~h}$ et $4 \mathrm{~h}$ du matin...) pour les femmes et les garçons de moins de 15 ans. La durée de la journée de travail ne pouvait excéder 12 heures. Les soins liés aux accidents du travail devaient être pris en charge par les employeurs. Dans les faits, bien peu de ces mesures furent mises en place en 1916. C'est sous l'impact des conflits sociaux des années 1920 que la plupart des employeurs durent se résoudre à appliquer la législation en vigueur $^{36}$.

34. Kawakami (2008 [1917] : 110).

35. Kawakami Hajime devint marxiste au cours des années 1920, fut expulsé de l'université de Kyoto en 1928 puis adhéra au PCJ en 1932. Arrêté en 1933, il refusa de se renier et passa le plus clair de son temps en prison à écrire de la poésie. Libéré en 1937, il se consacra à son autobiographie, qui devint l'un des grands succès de librairie de l'après-guerre, et à une nouvelle traduction du Capital de Karl Marx, restée inachevée. Il mourut, épuisé par les privations, en janvier 1946.

36. Imai (1998 [1974] : 93 sqq.). 


\section{La révolution russe vue du Japon}

La révolution russe, qui chassa le tsar en mars 1917 (révolution de Février), puis se radicalisa pour déboucher sur la prise du pouvoir par les bolcheviques début novembre (révolution d'Octobre), suscita, au Japon comme ailleurs, un immense intérêt parmi les intellectuels et les travailleurs. Dans la presse, les mots jadis réservés à la propagande socialiste - comme "capitalisme ", " socialisme ", " exploitation ", " classe sociale " - se retrouvaient désormais sous la plume de journalistes et d'essayistes. "Le mot de "révolution" était sur toutes les lèvres ${ }^{37}$ ". Dans son édition du 10 novembre 1917, le Tōkyō Asahi shinbun 東京朝日新聞 titra sur la prise du pouvoir en Russie par des "factions extrémistes d'ouvriers et de soldats». En quelques semaines, le mot extrémiste devint synonyme de bolchevique, et le terme restera longtemps pour qualifier le nouveau pouvoir russe. Pourtant, la proclamation par ce dernier du principe d'une paix sans annexions ni compensations et la dénonciation de la diplomatie secrète suscitèrent de grands espoirs parmi ceux qui comprenaient que les relations internationales fondées sur des rapports de force cyniques n'avaient fait que déboucher sur la catastrophe de la guerre. Mettre de l'ordre dans les relations entre nations nécessitait d'imaginer de nouvelles formes de coopération mondiale, et l'attitude des dirigeants bolcheviques suscita, dans un premier temps, de l'empathie dans une grande partie de l'opinion publique japonaise, y compris parmi les classes moyennes. Dans un article paru dans le Töyō keizai shinpō 東洋 経済新報 en juillet 1918 et consacré au "nouveau régime extrémiste russe ", le journaliste Ishibashi Tanzan 石橋湛山 (1884-1973) rappelait que les " troubles » qui avaient éclaté dans ce pays étaient le produit d'une "guerre civile de classes, elle-même conséquence d'une situation économique ", et qu'on aurait tort de négliger ce que pense profondément le peuple russe et de le laisser à son sort. À l'encontre du sentiment du gouvernement japonais, Ishibashi concluait qu'il « fallait reconnaître le régime extrémiste russe et même l'aider ${ }^{38} »$.

37. Arahata (2016 [1975] : 384).

38. Ishibashi Tanzan, «Kagekiha o enjo seyo » 過激派を援助せよ (Aidons les factions extrémistes), Tōyō keizai shinpō, 25 mars 1918, et “ Kagekiha seifu o shōnin seyo " 過激派 政府を承認せよ (Reconnaissons le gouvernement extrémiste), ibid., 25 juillet 1918, 
L'émergence du régime bolchevique eut évidemment de lourdes conséquences sur les rapports de force entre grandes puissances en Asie. Inquiets des effets sociaux et politiques de l'effondrement du pouvoir tsariste, les dirigeants japonais estimaient le nouveau régime communiste inacceptable. La convention russo-japonaise d'amitié, renouvelée en 1916, fut rapidement dénoncée par Tokyo. Pour certains commentateurs, l'effondrement de l'ancien régime en Russie faisait craindre qu'il n'advienne quelque chose de semblable au Japon, avec une remise en cause de la monarchie impériale. Mais d'autres - une partie des cadres de l'armée et certains milieux d'affaires - y voyaient une occasion en or pour avoir les mains libres en Mandchourie du Nord et en Sibérie. La révolution victorieuse à Pétrograd pouvait bien signifier une extension possible de la sphère d'influence japonaise et, en définitive, une bonne opportunité pour ceux qui rêvaient d'expansion sur le continent.

Inquiets néanmoins des premiers succès de l'Armée rouge, les Occidentaux, et notamment Washington, pressèrent le Japon, à partir de mai 1918, de venir en aide aux 50000 prisonniers tchécoslovaques de Sibérie qui avaient profité du chaos pour se constituer en armée anti-bolchevique. Il fut finalement décidé le 20 juillet que le Japon interviendrait militairement en Sibérie, avec l'appui américain, tandis que les alliés ouvriraient un nouveau front, plus tard, en Ukraine, à partir de la Mer Noire ${ }^{39}$. L'intervention japonaise aurait pour objectif de constituer un régime contrerévolutionnaire dans l'Extrême-Orient sibérien et de placer la Mandchourie $\mathrm{du}$ Nord sous influence japonaise, tout en pesant plus fortement sur le gouvernement chinois, lui-même fort inquiet de la pression communiste sur ses frontières septentrionales. Le départ du premier contingent japonais pour Vladivostok fut fixé au 14 août 1918.

reproduits dans Imai (1998 [1974]). Sur Ishibashi Tanzan, voir en français Joël Piguet dans Souyri (2014: 67-72). Il est intéressant de noter qu'Ishibashi Tanzan, devenu Premier ministre en 1956-57, fut alors partisan d'une reconnaissance immédiate du régime communiste chinois de Pékin. Cette reconnaissance n'interviendra qu'en 1972. 39. Fin 1917, les Alliés envoyèrent des troupes à Arkhangelsk. Fin 1918, des troupes françaises débarquèrent en Russie du Sud. Ces opérations, mal organisées et mal coordonnées, débouchèrent sur des échecs. 


\section{« La jacquerie des femmes de l’Etchū »}

Or, au cours de l'année 1918, la bonne conjoncture liée à l'expansion économique avait déclenché des phénomènes inflationnistes difficiles à maîtriser. Hausse démographique ${ }^{40}$, amélioration du niveau de vie des couches moyennes urbaines, prolétariat industriel dont les salaires repartaient à la hausse, allaient dans le sens d'une élévation de la demande, notamment en riz. Le système de la propriété foncière ne poussait cependant guère ni à une augmentation de la production ni à des gains de productivité dans la riziculture. En fait la production agricole stagnait. L'offre restait inférieure à la demande. En prévision de la montée des prix, les marchands de riz stockèrent. Malgré l'inquiétude déclarée des dirigeants, dont certains craignaient une explosion de type russe, aucune mesure particulière ne fut prise pour améliorer le sort des populations.

De leur côté, les autorités réquisitionnaient le peu de riz qui restait sur le marché pour équiper les soldats sur le départ vers la Sibérie. Le prix du riz se mit à flamber entre avril et juillet puis explosa début août ${ }^{41}$. À l'inquiétude devant la hausse des prix succéda soudain une grande anxiété. Dans les milieux populaires, on redoutait la faim. L'annonce que le gouvernement s'apprêtait à envoyer des troupes en Sibérie quand le peuple était au bord de la disette survenait on ne peut plus mal.

Le premier incident sérieux éclata le 23 juillet à Uozu (département de Toyama), un port de la mer du Japon. Là, le prix du riz avait augmenté de $35 \%$ depuis janvier. Une cinquantaine de femmes de marins se rassemblèrent sur le port et entravèrent le chargement d'un bateau transportant du riz en direction de Nemuro pour les soldats en partance. La police dispersa les manifestantes mais la presse rapporta l'incident. Des mouvements de même type impliquant des femmes éclatèrent les jours suivants dans les autres ports de la région, à Mizuhashi et à Namerikawa notamment. Au large de ces ports, les eaux étaient peu poissonneuses et la plupart des hommes étaient sur les bateaux, en pleine campagne de pêche, vers

40 La population totale du Japon passe d'environ 44 millions d'habitants en 1900 à 55 millions en 1920 .

41. En avril 1917, le prix du koku de riz est de 20 yens. En avril 1918, il est déjà de 33 yens, 40 yens à Osaka début août, 53 yens le 9 août. 
Hokkaidō ou Sakhaline. Les femmes, seules à la maison, s'inquiétaient de la hausse des prix. Cette population urbaine de pêcheurs était d'autant plus vulnérable qu'elle était totalement coupée de la paysannerie de l'arrièrepays, et ne pouvait guère compter sur des revenus d'appoint ${ }^{42}$.

À partir du 3 août, des cortèges de femmes se formèrent dans plusieurs agglomérations de la région. Les manifestantes réclamaient le riz « à sa juste valeur " (tekisei kakaku 適正価格), l'aide aux plus démunis et l'interdiction des exportations. Des affichettes appelaient la foule (qui se fit de plus en plus masculine) à se rendre à la nuit tombée au sanctuaire local. On faisait sonner le gong comme un tocsin puis on déboulait en cortège devant les maisons des accapareurs de riz, on brisait les réverbères, coupait les fils télégraphiques et, dans l'obscurité totale à l'exception des torches apportées par les manifestants, les maisons étaient prises d'assaut et démolies (uchikowashi 打ち壊し ${ }^{43}$. Si la police intervenait, elle était accueillie par des jets de tuiles et de pierres. On s'emparait des sacs de riz et parfois même on jetait le riz depuis le toit sur la foule ("Il pleut du riz, comme c'est drôle !») ${ }^{44}$. Les manifestants scandaient washira zenbu ga ichimi yazo わしら全部が一味やぞ45 (tous ensemble, tous ensemble !), reprenant cette expression ichimi tout droit sortie des révoltes des temps médiévaux ${ }^{46}$. Dès le 25 juillet, le Hokuriku Times 北陸夕イムス titrait : "l'ikki à l'assaut des maisons de marchands de riz ». Plusieurs journaux évoquèrent début août " la jacquerie des femmes de l'Etchū »(Etchū nyobō ikki 越中女房一揆), de l'ancien nom de la province qui correspondait au département de Toyama, et la presse nationale relaya l'information. Ces mouvements ressemblaient en effet à s’y méprendre aux poussées de fureur populaire de la première moitié du $\mathrm{XIX}^{\mathrm{e}}$ siècle, à l'époque des Tokugawa, quand on envahissait et démolissait les maisons des usuriers et des marchands ${ }^{47}$. La presse ne manqua pas de

42. Ces bourgades avaient une forte densité. Uozu comptait 15000 habitants, Mizuhashi 7000 et Namerikawa 10000 (Komatsu 2009 : 166).

43. Komatsu (2009: 167).

44. Komatsu (2009: 168).

45. Tachibana $(2014: 80)$.

46. La formule ichimi doshin一味同心 signifie « les cœurs à l'unisson ». Elle se retrouve dans les serments médiévaux par lesquels les membres d'un ikki juraient d'agir tous ensemble, à l'unisson (Katsumata : 2011).

47. Ninomiya (1990 : 391 sqq.). Voir aussi Katsumata (2011 : 249 sqq.). 
le relever, d'autant que la région avait connu de nombreux incidents à l'époque Meiji ${ }^{48}$. Entre le 23 juillet et le 19 août, on ne compta pas moins de vingt-deux nuits d'émeutes dans la région, regroupant selon le cas entre quelques dizaines et un millier de personnes. Ainsi la police rapporta, pour la seule bourgade de Namerikawa, trois cents manifestantes le 5 août, plus d'un millier d'hommes et de femmes le 6 août, sept cents personnes le 7, et six cents personnes manifestant dans deux endroits différents de la ville, soit mille deux cents personnes en tout, le 8 août ${ }^{49}$. À chaque fois, les femmes semblent avoir lancé le mouvement, qui gagne en puissance avec le renfort d'éléments masculins, ceux-ci attisant la colère et la violence de la foule. Parmi les hommes qui participèrent aux manifestations, le petit peuple: ouvriers des chantiers, journaliers, marchands colporteurs, rejoints parfois par des gens à peine plus aisés, petits commerçants, ouvriers imprimeurs, vanniers, voire employés de commerce... À chaque fois, le même scénario : on se rassemble devant la maison d'un spéculateur ou considéré comme tel (le 6 août à Namerikawa, la foule regroupée devant la maison d'un grossiste, Kanegawa Sōsaemon, comptait près de 2000 personnes selon les témoins, les deux tiers étant des femmes ${ }^{50}$ ). On fait le siège du grenier, on menace de brûler le bâtiment voire d'en massacrer les occupants (notons néanmoins que nulle part cette dernière menace ne fut mise à exécution).

Mais une autre inquiétude devint perceptible dont la presse se fit aussi l'écho. Le Toyama kyūhōo 富山急報 le nota dans son édition du 7 août :

La jacquerie des femmes de l'Etchū fait penser évidemment aux émeutes de la faim qui ont éclaté en février de l'an dernier dans les villes russes ${ }^{51}$.

48. Notamment des émeutes dites «bandori " (bandori sōdō バンドリ騒動), de l'appellation locale du mino 萜, le manteau en paille de riz revêtu traditionnellement par les révoltés. Sur le costume des révoltés, voir aussi Katsumata (2011 : 166 sqq.). En 1890, des troubles éclatèrent à Namerikawa suite à une hausse du prix du riz combinée à des réquisitions de l'État pour l'armée, obligeant les notables locaux à opérer des distributions de riz. En 1912 de nouveau des troubles touchèrent la région. Ces derniers étaient dans la tête de tous les esprits en 1918 (Kamiya 1986 : 9 sqq.) (Katsuyama $2010: 2-10$ ) (Imoto et al. 2004: 25-41).

49. Katsuyama (2010:39).

50. Kamiya (2004: 150).

51. Cité par Imai (1998 [1974] : 175). 
Le même jour, le journal local de Takaoka, le Takaoka shinpō 高岡新報, était encore plus explicite, sur le mode lyrique :

La révolution russe a débordé du chaudron. Le militarisme allemand, lui aussi, sera tôt ou tard maudit et expulsé du chaudron. Les émeutes populaires qui ont éclaté à Nishi Mizuhashi, à Higashi Mizuhashi et à Namerikawa nous envoient un signal social effrayant. Même s'il a été facile pour la police de rétablir l'ordre, pourra-t-on pour autant calmer les esprits ${ }^{52}$ ?

\section{Les émeutes se généralisent}

Or, à partir du 8 août, le mouvement - dont la presse avait largement rendu compte - changea soudain de nature ${ }^{53}$. Circonscrit géographiquement aux ports de la Mer du Japon, et socialement pour l'essentiel aux femmes de pêcheurs, il s'étendit soudain aux villes de l'intérieur, commençant par celles de l'ouest du pays (des émeutes éclatèrent à Okayama, Wakayama, Kyoto, Osaka). À Nagoya, le 10 août, le bruit se répandit qu'un grand meeting se tiendrait le soir dans le parc de Tsurumai pour évoquer la lutte contre la hausse des prix, et plus de 10000 personnes se rassemblèrent, ouvriers, étudiants, employés de commerce, pour écouter les discours. La foule commença à jeter des pierres sur les cordons de policiers, puis décida de s'en prendre aux maisons de commerce qui stockaient le riz, et l'émeute commença. Le lendemain soir, 50000 personnes se rassemblèrent au parc de nouveau, selon la police, et le lendemain encore 30 000. Le 11 août, les troubles gagnèrent Kōbe et le lendemain, les ouvriers des chantiers navals Mitsubishi provoquèrent le chaos dans l'usine : le soir, la ville était livrée à l'émeute.

L’envoyé spécial du journal Tökyō nichi nichi shinbun 東京日々新聞 témoigne :

Quand la nuit tomba, la ville de Kōbe était en proie à une gigantesque émeute. Dans l'avenue de Sakaemachi, un, deux, trois groupes, plus encore, marchaient en vociférant. La maison de commerce Suzuki, sise à Sakaemachi yonchōme, était en flammes. Le bâtiment du Heishinkan, où se trouvent les bureaux d'un prêteur sur

52. Imai (1998 [1974]: 175).

53. Komatsu (2009: 165). 
gages réputé dur avec le petit peuple, brûlait également, ainsi que les entrepôts de Hyōgo de raffinage du riz qui appartiennent à la maison Suzuki. Les trois étages du bâtiment du Kōbe shinbun, le journal de Kōbe qui se fait souvent l'avocat des intérêts Suzuki, furent incendiés. Les aciéries de Wakinohama étaient en cendres ainsi que le siège de la caisse des dépôts de Kōbe. L'incendie toucha aussi le siège principal de la maison Suzuki, au Grand Hôtel, au milieu des cris et des insultes de la foule.

Le lendemain, le 13 août au matin, je pris un pousse-pousse pour faire le tour de la ville et nous fûmes retenus par un groupe de gens qui nous bloquèrent le passage. Un homme à l'air convaincu qui semblait être le chef, nu avec un linge en coton autour de la taille et un sabre fourré autour des hanches, s'en prit à moi qui étais perché en haut de la voiture : "Quand nous sommes en train de mater les salopards qui s'en prennent à notre existence, toi tu te balades en voiture ? C'est honteux. Descends de là !”

Je me rendis alors au parc de la Minato où, devant un sanctuaire entouré d'arbres, avait lieu un meeting improvisé. Des hommes, un bandeau ceint autour du front, vêtus d'une veste à rayures blanches, criaient des slogans : "À bas le cabinet Terauchi ! Salauds de riches! Cette nuit, on va aller chercher le riz que vous avez caché c $^{4}$ !»

Dans la plupart des villes du Kansai, à Kyoto notamment, les gens des ghettos de discriminés (buraku 部落) jouèrent un rôle important, à côté de personnes issues de milieux populaires divers, exaspérées par la hausse des prix. À Fukui, la foule attaqua la préfecture et un commissariat. Puis le 13 août, les émeutes gagnèrent Tokyo, le Kantō et le Kyūshū. Les forces de l'ordre tirèrent sur la foule pour disperser les manifestants. De nombreuses villes japonaises connurent des scènes d'émeute au cours de ces journées. En certains endroits, les danses de la fête du Bon, la fête des morts, le 15 août, dégénérèrent et les danseurs se muèrent en émeutiers, comme à Kōga, dans le département de Shiga ${ }^{55}$. Passé la mi-août, le mouvement gagna les villages de l'intérieur ainsi que les agglomérations minières comme Miike dans le nord de Kyūshū. En août 1918, on dénombrait 108 usines en grève, un record! Des troubles éclatèrent jusqu'à Séoul, le 28 août, contre la hausse des prix du riz ${ }^{56}$. Tout le pays, sauf Okinawa, le nord de Honshū et Hokkaido ${ }^{57}$, fut touché par ce mouvement d'une ampleur tout à fait

54. Reproduit dans Imai (1998 [1974] : 177-178).

55. Komatsu (2009: 170).

56. Narita (2007: 88).

57. On y rapporte toutefois quelques incidents mineurs, comme à Hakodate le 18 août. 
inconnue jusqu'alors. Le 12 septembre, les mineurs de Manda, à Miike, étaient quasiment en état d'insurrection.

Le retournement de la conjoncture contribua à calmer les esprits à partir de la fin août. Pris de panique devant l'ampleur de l'émeute, les marchands jetèrent sur le marché les stocks qu'ils détenaient, faisant redescendre rapidement le prix du koku de riz à des niveaux plus raisonnables. À la mi-septembre, les prix étaient revenus aux niveaux de 1916. Mais les émeutes s'étaient succédé à un rythme quasi ininterrompu pendant près de six semaines. Débordée, la police fut remplacée par l'armée dès le 11 août à Kyoto. À Kure, l'infanterie de marine fut mobilisée contre la foule le 18 août, faisant quatre morts ${ }^{58}$. Environ 100000 hommes de troupe participèrent à la répression des émeutes. On releva en tout une trentaine de tués, ce qui est finalement assez peu, eu égard à la violence déployée. Plusieurs milliers de personnes furent arrêtées, sept cents d'entre elles furent condamnées à des peines de prison, dont soixante-et-onze à des peines de travaux forcés. Deux condamnés à mort furent exécutés (issus du ghetto des burakumin de Wakayama). La plupart des personnes arrêtées et condamnées étaient des hommes jeunes, issus des milieux les plus humbles de la société.

De nombreux contemporains, ne pouvant s'expliquer l'ampleur des émeutes, soupçonnèrent un complot organisé5 ${ }^{59}$. Le mouvement avait pourtant éclaté sans leaders, sans organisation, de manière tout à fait spontanée. Les socialistes ou les syndicalistes n'y eurent aucune influence notable. On a vu que les femmes jouèrent un rôle central dans les premières manifestations. Dans les grandes villes, c'est le petit peuple qui entra en action : prolétaires des petits métiers, ouvriers des usines, parias des quartiers discriminés, mineurs, métallos des aciéries, travailleurs coréens puis, plus tard, quand le mouvement toucha les campagnes, petits paysans qui s'en prirent aux propriétaires fonciers.

Le gouvernement commit par ailleurs une erreur stratégique en cherchant à museler la presse pour l'empêcher de relater l'importance des événements. Dès le 7 août, le Takaoka shinpō 高岡新報, jugé trop en empathie avec les manifestants, fut censuré. Le 14, le gouvernement interdit à la

58. Komatsu (2009: 166).

59. Eguchi (1989 : 74), Kinbara (2009 [1973] : 19-20). 
presse nationale de rapporter l'ampleur des émeutes. Il est vrai que les journalistes utilisaient un métalangage en évoquant le mot $i k k i$ ou les pratiques d'uchikowashi (démolissage) qui résonnaient dans les oreilles populaires et étaient parfaitement compréhensibles. Rendus furieux par une censure brutale sur l'information, les patrons de presse et les journalistes organisèrent dès lors réunions sur réunions, à Tokyo, dans le Kansai et ailleurs en province, pour protester. La plupart d'entre eux avaient déjà participé au mouvement contre le cabinet Katsura en 1913. "Les émeutes ne sont pas le fait de la presse mais des erreurs du gouvernement ", proclamaient de nombreuses motions de journalistes votées en assemblée. Une grande partie des journaux mena alors campagne contre le gouvernement Terauchi, accusé d'inertie, qui devait "désormais tirer les conséquences de ses erreurs ${ }^{60}$ ". La bataille pour la baisse du prix du riz se doubla dès lors d'une bataille pour la liberté d'expression, largement inspirée des thèses de Yoshino Sakuzō. Dès septembre, Ishibashi Tanzan note que ces émeutes seraient inévitables tant que le fonctionnement politique serait aussi sclérosé. Pour lui, les couches prolétariennes n'avaient fait que chercher à se défendre et les émeutes étaient donc le reflet de la gravité de la crise politique ${ }^{61}$. De son côté, le socialiste Arahata Kanson 荒畑寒村 (1887-1981) explique dans ses mémoires que "face à un peuple qui crevait de faim, le gouvernement n'a pas pris la moindre mesure et d'ailleurs, s'il en avait prise une, elle aurait été probablement d'un effet mineur. Le peuple a donc pris les choses en main $^{62}$ ".

Devant l'ampleur de la crise et l'hostilité de la presse, le gouvernement Terauchi fut contraint à la démission le 21 septembre. Hara Takashi 原敬 (1856-1921), un dirigeant de parti parlementaire non issu des anciennes coteries féodales du Sud-Ouest, devint chef de gouvernement. C'était là une première et un coup sévère porté à l'oligarchie politique qui présidait aux destinées du pays depuis la restauration monarchique ${ }^{63}$. De leur côté, les populations japonaises n’avaient guère apprécié de voir l'armée

60. Imai (1998 [1974] : 182-183).

61. Ishibashi Tanzan, "Sōshō no seijiteki igi 争訟の政治的意義 " (La signification politique de la contestation), Töyō keizai shinpō, 5 septembre 1918 (Ishibashi 1971 : 74-78).

62. Arahata (2016 [1975]: 388).

63. Eguchi (1989 : 59-60) ; Kinbara (2009 : 93). 
intervenir contre elles. On peut lire dans l'éditorial du 22 août de l'Ōsaka Asahi shinbun:

Jusqu'à présent, quand l'État utilisait la force dans les affaires internationales, le peuple japonais approuvait avec frénésie les mouvements de troupes à l'étranger, comme si les territoires conquis allaient lui appartenir. Or désormais il ne s'intéresse plus aux opérations extérieures qui ne semblent plus le concerner. Quand les autorités politiques évoquent un moment capital qui se joue sur les théâtres d'opération à l'étranger, le peuple ne se demande plus si ce sera utile au pays. Chacun s'interroge plutôt sur l'utilité de tout cela pour son profit personnel ${ }^{64}$.

\section{Premières réflexions sur la crise}

Les observateurs qui se sont alors penchés sur cette crise y virent la conjonction de plusieurs mouvements. D’abord une anxiété populaire devant les difficultés croissantes à assurer la stabilité du foyer alors que l'inflation dérapait et que le gouvernement, préoccupé par l'expédition de Sibérie, restait inerte. C'est ce qui fut à l'origine de la colère des femmes de pêcheurs notamment. Dans les villes, le petit peuple se révolta contre «l'immoralité des riches " qui continuaient de spéculer sur la misère des bonnes gens. Alimenté par l'inflation, perçue comme une manœuvre pour s'enrichir encore sur leur dos, le ressentiment contre les nantis joua certainement un rôle essentiel. L'absence de systèmes d'aide laissa les plus pauvres démunis face à la flambée des prix, et l'inexistence de moyens pour contrecarrer la crise fut comprise comme une trahison de la nécessaire bienveillance que devaient exercer les plus puissants à l'égard des plus faibles, dans le cadre de représentations générales aux accents toujours confucianistes. Les puissants avaient en quelque sorte failli à leur devoir de protection et, dans ces conditions, la violence paraissait légitime. On fit prévaloir une « économie morale " et un certain sens de la justice, préoccupations qui résonnaient comme en adéquation avec les thèses de Kawakami Hajime, rappelées ci-dessus. À la question des autorités qui demandaient aux manifestants

64. Cité par Imai (1998 [1974] : 181). L'expédition en Sibérie, décidée dans le secret des états-majors sans véritable débat national, fut considérée dans l'opinion avec une grande froideur. Les bulletins militaires tombaient dans l'indifférence, voire l'hostilité. 
arrêtés s'ils ne regrettaient pas d'avoir participé aux émeutes, nombreux furent ceux qui répondirent : "Mais pourquoi donc devrais-je le regretter ? C'est cela qui a fait baisser le prix du riz, non ${ }^{65}$ ?"

L'intervention des gens des ghettos, les descendants des parias, qui vinrent grossir les rangs des émeutiers, notamment dans les villes du Kansai, était évidemment liée à la hausse des prix mais ces gens étaient aussi exaspérés par un sentiment général et de plus en plus insupportable de ne pas être traités comme les autres. Le mouvement des mineurs et des petits paysans - qui se propagea alors que les prix avaient commencé à redescendre - traduisit aussi une exaspération contre les conditions de travail difficiles auxquelles ils étaient réduits. Les tensions sociales qui éclatèrent au grand jour en septembre relevaient déjà d'une autre dimension qui dépassait la lutte contre la cherté. Gotō Shinpei 後藤新平 (1857-1929), alors ministre des Affaires étrangères, pensait qu'il s'agissait là d'une manifestation extrême de la guerre des classes ${ }^{66}$. Après avoir été paralysés par l'atmosphère de "l'ère d'hiver ", ouvriers, mineurs et petits paysans redressaient en effet la tête. Leur mouvement préfigurait l'agitation sociale qui caractérisera la nouvelle période $^{67}$.

Au-delà des événements eux-mêmes, la violence qu'exprimait le mouvement renvoyait - et nombreux furent les analystes de l'époque qui le notèrent - à une incapacité profonde du système politique japonais d'alors à prendre en compte les aspirations des couches sociales subalternes, qui se sentirent abandonnées par l'État. Certains, comme les démocrates Yoshino Sakuzō ou Ishibashi Tanzan, accusèrent un gouvernement incapable d'être à l'écoute du peuple et pointèrent du doigt la bureaucratie d'État et le Parlement, totalement dénués, l'un comme l'autre, de la moindre volonté de comprendre les difficultés populaires et de leur trouver une solution. Fukuda Tokuzō 福田徳三 (1874-1930) pensait que l'imprévoyance du gouvernement avait poussé le peuple "à un point extrême " où le droit à la vie l'emporte sur le droit de propriété68. Au bout du compte, l'aggravation sensible des divisions sociales, la distribution inégale de la richesse,

65. Komatsu (2009: 168).

66. Gotō Shinpei dans son journal personnel, cité par Kinbara (2009 : 19).

67. Gordon (1991: 108).

68. Cité par Duus \& Scheiner (1998: 177). 
les difficultés économiques combinées à l'impéritie des classes possédantes, avaient poussé le peuple dans la rue ${ }^{69}$. Le socialiste Katayama Sen 片山潜 (1859-1933), à l'époque en exil aux États-Unis, cherchant à saisir le sens historique de l'épisode, y voyait la première étape de la révolution à venir. Plus tard, il écrira depuis Moscou, en 1933 :

Les classes laborieuses au Japon ont consenti de lourds et terribles sacrifices lors des émeutes du riz. Mais le mouvement de 1918, c'est la première action de lutte des masses qui a fait trembler les classes exploiteuses de ce pays ${ }^{70}$.

À l'automne 1918, la capitulation d'un ministre conservateur, incapable de réagir à une crise sociale, donna de l'espoir à tous ceux qui luttaient contre un système qui ne laissait guère de place à la libre expression et à la contestation. Tous ceux qui, dans les classes moyennes, s'étaient opposés les années précédentes à un système politique verrouillé par les anciennes cliques féodales des principautés du Sud-Ouest, le Conseil privé de l'empereur (Sūmitsuin 枢密院), les Anciens (genrō 元老) et les factions militaires, retrouvèrent espoir. Certes, les émeutes du riz ont pu être décrites comme la conséquence d'une grave mais classique crise de subsistance que les structures modernes ne permettaient pas de juguler, là où les mécanismes traditionnels seigneuriaux de régulation sous les Tokugawa se révélaient relativement efficaces. Mais dans les grandes villes comme à Tokyo, les émeutiers ne s'en prirent pas qu'aux maisons des spéculateurs, ils jetèrent des pierres sur les grands magasins de Nihonbashi, sur les boutiques de vêtements chics de Ginza, sur les restaurants, les banques et les bâtiments des grandes entreprises qui incarnaient l'enrichissement brutal de certains, bref s'en prirent à tout ce qui représentait ce luxe des couches moyennes et supérieures auxquels les manifestants n'avaient pas accès. Et comment ignorer, dans la foulée de ces émeutes, la tenue d'un congrès national des syndicats en août 1919 et la naissance de groupuscules prolétariens dont certains seront à l'origine du Parti communiste ? Le rôle remarqué dans les manifestations des discriminés issus des ghettos de buraku est souvent considéré comme le premier pas qui mena à la naissance de l'organisation des parias, la Suiheisha 水平社,

69. Ibid.

70. Katayama (1963 [1933] : 453). 
fondée en $1922^{71}$. Enfin, le rôle des femmes dans le déclenchement des émeutes intervint dans un contexte de contestation grandissante de la position subordonnée des femmes dans la société ${ }^{72}$, même si on se rend compte aujourd'hui qu'elles ont toujours joué un rôle important dans les révoltes sous l'ancien régime Tokugawa ${ }^{73}$. Nombreux furent les observateurs de l'époque qui comprirent que les "émeutes du riz " n'étaient pas qu'une fureur populaire de type classique liée à une crise de subsistance, mais qu'il s'agissait d'une crise politique, sociale, économique de grande ampleur qui, d'une certaine manière, contribuait à " délivrer une société bloquée " par un couvercle de plomb, celui de la répression contre le mouvement social ${ }^{74}$.

\section{$* * *$}

L'année 1918 marque, à n'en pas douter, un jalon dans l'histoire de l'archipel, à l'origine d'un nouveau cycle de contestations et de ruptures : le Japon n'est plus seulement une société engagée dans un processus de modernisation, qui progresse avec plus ou moins de bonheur. L'ambiguïté du mouvement, à la fois fureur populaire de type classique et mouvement social ancré dans la réalité du monde capitaliste, est à l'image du Japon de cette époque, où cohabitent des modes de pensée qui s'inscrivent tout autant dans le vocabulaire et l'idéologie "à la chinoise " que dans des discours cherchant à réguler les systèmes d'exploitation, pour les améliorer ou les dépasser. Les émeutes du riz sont un tournant non seulement parce qu'elles correspondent à l'irruption d'une conflictualité sociale sans

71. Matsuo Takayoshi, cité par Meyer (2005 : 70-71).

72. Sur ces questions, voir en français Lévy (2014) et Souyri (2016:431-455).

73. Une synthèse sur le rôle des femmes dans les émeutes d'ancien régime par Tsutsumi Yōko dans Hosaka (2000). Voir également, pour les révoltes de femmes dans l'Etchū, Katsuyama (2010).

74. On notera que l'épisode inflationniste de 1918 touche de nombreuses contrées en Asie : la Corée en est aussi victime et des incidents éclatent à Séoul fin août contre le prix excessif du riz, alors que l'Indochine française, la Birmanie britannique et le royaume du Siam cherchent à acquérir du riz sur les marchés extérieurs, contribuant à tendre les prix (Narita 2007 : 88). Sur les émeutes du riz, la grande étude de référence reste Inoue \& Watanabe (1962). Voir aussi Rekishi hyōron (1968). 
commune mesure avec ce qui était connu jusqu'alors, et qui se manifestera pendant toutes les années 1920, mais parce qu'elles indiquent aussi la fin d'un cycle de révolte, celui de l'émeute spontanée et brutale qui marqua toute l'histoire du Japon au Xix ${ }^{e}$ siècle. Le nouveau cycle qui commence alors traduit tout autant l'émergence d'une classe ouvrière combative que l'essor d'une société de masse avec une nouvelle culture de la consommation (taishü bunka 大衆文化), ou encore de nouvelles couches intellectuelles d'une génération passée par les lycées et les universités ${ }^{75}$, c'est-à-dire éduquée dans le cadre d'un système de pensée largement différent de celui qui forma la génération précédente.

Le Japon se trouve dès lors impliqué, comme les autres pays avancés de son temps, dans une crise des relations sociales dont la résolution se pose en termes de plus en plus antagoniques. La montée du mouvement ouvrier à partir des années 1918-1919, la crise de l'économie qui frappe le système en 1930, l'impérialisme et l'intervention de l'État comme remèdes à la crise, les tendances autoritaires de plus en plus affirmées dans le corps social puis finalement la guerre, sont des processus que connaissent nombre de sociétés avancées dans les années 1920 et 1930. Le Japon n’y échappe pas. En ce sens, il n'est plus un pays engagé dans la modernisation de ses structures, il est déjà un pays « moderne » confronté à une crise générale de la « modernité ».

75. Le nombre d'étudiants est de 24000 en 1900, de 47000 en 1910, de 80000 en 1920. À partir de 1918-1919, on commence à évoquer l'existence d'un "mouvement étudiant". 


\section{Bibliographie}

ARAHATA Kanson 荒畑寒村 2016 [1975] Kanson jiden 寒村自伝 (La vie de Kanson par lui-même), t. 1, Tokyo, Iwanami shoten 岩波書店.

\section{CRAWCOUR E. Sidney 1997}

« Industrialisation and Technological Change, 1885-1920 », in Yamamura Kozo (ed.) The Economic Emergence of Modern Japan, Cambridge, Cambridge University Press : 50-115.

\section{DUUS Peter \& SCHEINER Irwin 1998} «Socialism, Liberalism, and Marxism, 1901-1931 », in Bob T. Wakabayashi (ed.) Modern Japanese Thought, Cambridge, Cambridge University Press: 147-206.

\section{EGUCHI Keiichi 江口圭一 1989}

Futatsu no taisen 二つの大戦 (Les deux grandes guerres), Tokyo, Shōgakkan 小学館, coll. «Taikei Nihon no rekishi » 大系日本の歴史， 14.

\section{GARON Sheldon 1987}

The State and Labor in Modern Japan, Berkeley, University of California Press.

\section{GORDON Andrew 1991}

Labor and Imperial Democracy

in Prewar Japan, Berkeley,

University of California Press.

\section{HAMON Claude 2007}

Shibusawa Eiichi (1840-1931), Bâtisseur du capitalisme japonais, Paris, Maisonneuve \& Larose.
HOSAKA Satoru (dir.) 保坂智 2000 Ikki to shūen一揆と周緑 (Les révoltes et les marges), Tokyo, Aoki shoten 青木 書店, coll. « Minshū undō shi »民衆 運動史 (Histoire des mouvements populaires), 1.

IMAI Seiichi 今井清— 1998 [1974] Taishō demokurashii 大正デモクラシー (La démocratie de l'ère Taishō), Tokyo, Chūō kōron sha 中央公論社, coll. « Nihon no rekishi 日本の歴史», 23.

IMOTO Mitsuo 井本三夫 et al. (dir.) 2004 Zusetsu Kome sōdō to minshushugi no hatten 図説米騒動と民主主義の発展 (Les émeutes du riz et le développement de la démocratie), Tokyo, Minshūsha 民衆社.

\section{INOUE Kiyoshi 井上清}

\& WATANABE Tōru 渡部徹 (dir.) 1962 Kome sōdō no kenkyū 米騒動の研究 (Recherches sur les émeutes du riz), t. 5, Tokyo, Yūhikaku 有斐閣.

\section{ISHIBASHI Tanzan 石橋湛山 1971} Ishibashi Tanzan zenshū 石橋湛山全集 (CFuvres complètes), t. 2, Tokyo, Tōyō keizai shinpō sha 東洋経済新報社.

ISHIKAWA Takuboku 石川啄木 1969 Ishikawa Takuboku shū 石川啄木集 (Euvres complètes d'Ishikawa Takuboku), Tokyo, Kadokawa shoten 角川書店, coll. « Nihon kindai bungaku taikei »日本近代文学大系, 23.

KAMIYA Nobuo 紙谷信雄 1986 Namerikawa machi ni okeru minponshugi no tenkai 滑川町における民本主義の展開 
(L'essor de la démocratie dans la ville de Namerikawa), Uozu (à compte d'auteur).

KAMIYA Nobuo 紙谷信雄 $\mathbf{2 0 0 4}$

Kome sōdō no rironteki kenkyū 米騷動の 理論的研究 (Recherches théoriques sur les émeutes du riz), Uozu, Kakimarusha 柿丸舎.

\section{KATAYAMA Sen 片山潜 1963} « Nihon ni okeru sen kyūhyaku jūhachi nen no kome sōdō no jūgo shūnen ni yosete »日本における一九一八年の米騒動 の一五周年によせて (Les émeutes du riz de 1918, quinze ans après) [Communist International, 1933], in Ōkouchi Kazuo (dir.) 大河内一男, Shakaishugi 社会主義 (Socialisme), Tokyo, Chikuma shobō 筑摩書房, coll. « Gendai Nihon shisō Taikei » 現代日本思想大系, 15.

\section{KATSUMATA Shizuo 勝俣鎮夫 2011} Ikki, Coalitions, ligues et révoltes dans le Japon d'autrefois, trad. Pierre-François Souyri, Paris, CNRS éditions, [éd. or. Ikki 一揆, Tokyo, Iwanami shoten 岩波書店, 1982].

\section{KATSUYAMA Toshiichi 勝山敏一 2010 Onna ikki no tanjō 女一揆の誕生 (La naissance des révoltes de femmes), Toyama, Katsura shobō 桂書房.}

KAWAKAMI Hajime 河上肇 2008 [1917] Binbō monogatari 貧乏物語 (Roman de la misère), postface d'Ōuchi Hyōe 大内兵衛, Iwanami bunko, Tokyo, Iwanami shoten 岩波書店.

KINBARA Samon 金原左門 2009 [1973] Taishōki no seitō to kokumin. Hara Takashi naikakuka no seiji katei 大正期の政党と 国民一原敬内閣下の政治過程 (Les partis politiques et le peuple pendant l'ère Taishō : I'évolution politique du cabinet Hara), Tokyo, Hanawa shobō 塙書房.

KOMATSU Hiroshi 小松裕 2009

<Inochi> to teikoku Nihon「いのち」と帝国 日本 (La vie et l'empire du Japon), Tokyo, Shōgakkan 小学館, coll. «Zenshū Nihon no rekishi » 大系日本の歴史, 14 .

\section{LÉVY Christine 2002}

" Kōtoku Shūsui et l'anarchisme », Ebisu - études japonaises, 28 : 61-86.

\section{LÉVY Christine 2010}

«Autour de l'Affaire du crime de lèsemajesté ", dans Censure, autocensure et tabous, Actes du Quatrième colloque d'études japonaises de I'Université de Strasbourg, Arles, Philippe Picquier.

\section{LÉVY Christine (dir.) 2014}

Genre et modernité au Japon. La revue Seitō et la femme nouvelle, Rennes, Presses universitaires de Rennes.

\section{MEYER Harald 2005}

Die Taishô-Demokratie, Begriffsgeschichtliche Studien zur Demokratierezeption in Japan von 1900 bis 1920, Berne, Peter Lang.

\section{NARITA Ryūichi 成田龍一 2007}

Taishō demokurashii 大正デモクラシー (La démocratie de l'époque Taishō), Tokyo, Iwanami shoten 岩波書店, coll. «Shirīzu Nihon kingendaishi » シリーズ日本 近現代史, 4 . 
NINOMIYA Hiroyuki 1990

« L'époque moderne », in Francine

Hérail (dir.), Histoire du Japon,

Le Coteau, Horvath : 301-424.

Rekishi hyōron 歴史評論 1968

Kome sōdō, 50 nen 米騒動 50 年

(Les émeutes du riz, cinquante ans après), 216.

SOUYRI Pierre-François (dir.) 2014 Japon colonial 1880-1930. Les voix de la dissension, Paris, Les Belles-Lettres.

SOUYRI Pierre-François 2016

Moderne sans être occidental. Aux origines du Japon d'aujourd'hui, Paris, Gallimard.

\section{STEGEWERNS Dick 2003}

«Yoshino Sakuzo: The Isolated

Figurehead of the Taisho Generation », Nationalism and Internationalism in Imperial Japan. Autonomy, Asian Brotherhood, or World Citizenship, Londres, New York, Routledge \& Curzon.

TACHIBANA Yūichi 立花雄一 2014 Inpei sareta onna kome sōdō no shinsō 隠蔽された女米騒動の真相 (La vérité cachée sur les émeutes du riz de femmes), Tokyo, Nihon keizai hyōronsha 日本経済評論社.

YAMAKAWA Hitoshi 山川均 1976 《Minponshugi no hanmon » 民本主義 の煩悶 (La démocratie minpon shugi dans la tourmente) [14 mars 1918], Yamakawa Hitoshi shū 山川均集 (Fuvres de Yamakawa Hitoshi), Tokyo, Chikuma shobō 筑摩書房, coll. « Kindai Nihon shisō taikei » 現代日本思想大系, 19.

YOKOYAMA Gennosuke 横山源之助 1985 [1899]

Nihon no kasō shakai 日本の下層社会 (Le Japon d'en bas), Tokyo, Iwanami shoten 岩波書店.

YOSHINO Sakuzō 吉野作造 1984 [1916] «Kensei no hongi o toite sono yūshū no bi o nasu no michi o ronzu » 憲政の 本義を説いてその有終の美を成すの途を 論ず (Les principes du gouvernement constitutionnel et les moyens de les réaliser pleinement), in Mitani Ta.ichirō 三谷太一郎 (dir.), Yoshino Sakuzō 吉野 作造 Tokyo, Chūōkōronsha 中央公論社, coll. « Nihon no meicho »日本の名著， vol. 48 : 91-181.

\section{YOSHINO Sakuzō 1995}

«Fujin no seiji undō » 婦人の政治運動 (Le mouvement politique des femmes) [1915], in Yoshino Sakuzō zenshū 吉野 作造全集, vol. 3, Tokyo, Iwanami shoten 岩波書店 : 115-123. 